NOTICE: this is the author's version of a work that was accepted for publication in Knowledge-Based Systems. Changes resulting from the publishing process, such as peer review, editing, corrections, structural formatting, and other quality control mechanisms may not be reflected in this document. Changes may have been made to this work since it was submitted for publication. A definitive version was subsequently published in Knowledge-Based Systems, Vol.24, no.6 (August 2011). DOI: 10.1016/j.knosys.2011.02.005 


\title{
Semantic Service Matchmaking for Digital Health Ecosystems
}

\author{
Hai Dong, Farookh Khadeer Hussain \\ Digital Ecosystems and Business Intelligence Institute, Curtin University of Technology, Enterprise Unit 4, \\ De Laeter Way, Technology Park, Bentley, WA 6102, Australia
}

\begin{abstract}
The vision of Digital Ecosystems was initiated by the European Commission, with the purpose of constructing an information and communication technology environment to facilitate the sustainable development of small and medium enterprises. As a key subdomain of Digital Ecosystems, Digital Health Ecosystems provide crucial services to maintain the health of the main participants of Digital Ecosystems. We are concerned with the large-scale, ambiguous, heterogeneous, and untrustworthy health service information in Digital Health Ecosystems. An intensive survey found that current research cannot support accurate and trustworthy matchmaking between health service requests and health service advertisements in Digital Health Ecosystems. Therefore, in this paper, we propose a framework of a semantic service matchmaker, by taking into account the ambiguous, heterogeneous nature of service information in Digital Health Ecosystems. This framework is designed to make four major contributions, which are health service domain knowledge modeling, online health service information disambiguation, health service query disambiguation and health service quality evaluation and ranking. In order to thoroughly evaluate this framework, we implement a prototype - a Semantic Health Service Search Engine, and execute a series of experiments on the prototype using a functional testing and simulation approach.
\end{abstract}

Keywords: Digital Ecosystems, Digital Health Ecosystems, semantic search, semantic service matchmaking

\section{Introduction}

Digital Ecosystems is a research vision initiated in 2000 by the European Commission, under the auspices of the Lisbon European Council, aimed at developing a knowledge-based economy for sustainable economic growth of small and medium enterprises (SMEs) with the support of information and communication technologies (ICT) [12]. SMEs comprise $99.7 \%$ of companies in Europe and account for nearly half of the European Gross Domestic Product (GDP) (http://www.ueapme.org/), which are key drivers of European economy. Nevertheless, 93\% of SMEs have fewer than 10 employees, and these SMEs experience difficulties in adopting ICT to promote their economic growth due to the lack of financial and technical supports. Therefore, the European Commission proposed a technological infrastructure - Digital Business Ecosystems, by means of which services and information over the Internet can be discovered and linked to realize networked transactions, in order to enhance the knowledge creation and sharing between SMEs [37]. In 2006, Chang and West boosted Digital Ecosystems research by extending the bounds of Digital Ecosystems from the business domain to broader domains such as health, education etc. [8, 9]. Furthermore, from the perspective of services, Chang et al. defined the roles of species in a Digital Ecosystem - service providers and service requesters. In addition, species can flexibly switch between the two roles and even simultaneously play the two roles.

Our research is concerned with the issue of service matchmaking in a subdomain of Digital Ecosystems - Digital Health Ecosystems. Service matchmaking refers to a mechanism matching between a service request provided by a service requester and a service advertisement published by a service provider. In Digital Health Ecosystems, species and services are heterogeneous in nature; namely, species contain hospitals, pharmacies, clinics, health practitioners, and definitely individuals etc., and services refer to services provided or requested by these species [22]. Furthermore, there is substantive health service information (e.g. numerous online health service advertisements) in Digital Health Ecosystems, which is ambiguous and heterogeneous without sufficient supports for service discovery and annotation. The reason for our interest in this domain is that health services contribute to ensuring the health of the main participants within Digital Ecosystems - biological species (people) and economic species (organizations), which play a crucial role in the stabilization and sustainable development of Digital Ecosystems [14]. On account of the heterogeneity and ambiguity of large-scale service information, service requesters may not have enough knowledge regarding their service requests. Nowadays, there are a great 
number of systems designed for local health service searches. However, according to the existing surveys in Section 2.1, it is observed that current health service search engines cannot help a service requester to precisely retrieve a service provider by taking into account the heterogeneous and ambiguous nature of service information in Digital Health Ecosystems.

Semantic web is an ongoing project, the aim of which is to provide meaning to the data in the web. By means of this project, the web is able to understand people's or machines' requests to use web contents [3]. To realize this objective, many semantic web language standards and semantic web development tools have been developed, such as Web Ontology Language (OWL), Resource Description Framework (RDF), Resource Description Framework Schema (RDFS), SPARQL Protocol and RDF Query Language (SPARQL), Protégé etc. Ontology is a form of domain knowledge representation [21], which comprises concepts, properties of concepts and relations between concepts. Ontology is the backbone of the semantic web, being an efficient tool for representing knowledge and for knowledge sharing between people-people, people-machine and machine-machine. Ontology can be represented by the semantic web languages such as OWL, RDFS, RDF, etc.

Based on our survey of existing literature in Section 2.2, it is found that semantic web technologies have been widely utilized in service matchmaking in the web service field, with the purpose of improving the matching performance between a service request and a service advertisement. Nevertheless, none of the research focuses on semantic service matchmaking in Digital Health Ecosystems. The main difference between web services and Digital Health Ecosystem services is that service requests and service advertisements in the former are described by Web Service Description Languages (WSDL), and in the latter can be described by arbitrary natural languages. Hence, we can deduce that service matchmaking in Digital Health Ecosystems could face more challenges than it does in the web service field owing to the non-standardization of the former.

Analogous to web services, people are also concerned with the issue of trust or quality of online service advertisements. The quality of web services can be assessed by their non-functional properties (NFPs); however, the non-standard Digital Health Ecosystem service advertisements cannot be assessed in the same manner. Hence, it is necessary for us to find a way to assess the trustworthiness of Digital Health Ecosystem service advertisements during the service matchmaking process.

In this paper, to overcome the challenges of service matchmaking in Digital Health Ecosystems, we propose a framework for a semantic service matchmaker which takes into account the heterogeneous and ambiguous nature of services in Digital Health Ecosystems. This framework makes use of semantic web languages to specifically describe service domain knowledge in Digital Health Ecosystems, by means of using semantic service matchmaking and quality of services (QoS) to match health service requests with health service advertisements.

The rest of the paper is organized as follows: in Section 2, we conduct a general survey of the current semantic health search research and semantic service matchmaking research; in Section 3, we present an overview of our proposed solution; in Section 4, we introduce a health service ontology for modeling the Digital Health Ecosystem service domain knowledge, and a health service metadata schema to standardize online health service information; in Section 5, we present the framework and workflow of the proposed semantic service matchmaker, and explain the involved matchmaking algorithm and a service ranking methodology; in Section 6, we implement a series of experiments in order to validate the proposed framework; the conclusion is drawn and future work is planned in the final section.

\section{Related Work}

In this section, we undertake a general survey of health service search engines and semantic service matchmakers to explore the issues that arise when these are confronted by the ambiguous and heterogeneous nature of services in Digital Health Ecosystems.

\subsection{Semantic Health Search Engines}

With the emergence of the Internet technology, increasing health service requesters are accustomed to search for service information by the Internet. A survey conducted by Andreassen et al. [1], indicated that up to $71 \%$ of the Internet users in this survey had used the Internet for health purposes. Accordingly, various health information search engines have been developed to assist service requesters to precisely retrieve health information. Nevertheless, existing health information search engines meet the challenges of fulfilling the requirement of accurate search. In 
2001, Berland et al. [2] conducted a survey of 14 health information search engines and found that less than one quarter of the links on first pages returned by the search engines have any relevance to queries. There are two reasons for this problem:

1. User queries could be ambiguous, as a result of users not having enough health domain knowledge about their actual requests.

2. Search engines cannot understand the meanings of user queries as a result of a lack of semantic supports.

In order to resolve the above problems, several researches have been conducted in the field of semantic health information search for user query disambiguation. Can et al. [6] proposed a semantic search engine - MedicoPort for users with limited knowledge of the health domain, where domain knowledge is formulated using the Unified Medical Language System (UMLS). UMLS contains three parts - a UMLS SPECIALIST Lexicon, a UMLS semantic network and a metathesaurus. Through the SPECIALIST Lexicon, medical terms can be extracted from user queries; through the UMLS semantic network and the metathesaurus, user queries can be formulated by retrieving synonyms of extracted terms. Finally, documents relevant to formulated queries can be retrieved and ranked according to their relevance. Holi et al. [26] proposed a fuzzy view-based semantic search engine for a semantic e-health portal - HealthFinland. HealthFinalnd is a semantic publishing platform allowing users to collaboratively create semantic health information [27]. The authors employed a fuzzy approach to map between ontology conceptannotated documents and search categories/views in the HealthFinland. They employed the FinMeSH - the Finish translation of Medical Subject Headings (MeSH) as the annotation ontology. Later, Suominen et al. [43] proposed a user-centric faceted search engine for the HealthFinland, in which several ontologies are employed by the authors, such as the $\mathrm{MeSH}$, the Finish General Upper Ontology (YSO) and the European Multilingual Thesaurus on Health Promotion (HPMULTI).

From the survey above, we found two limitations with the current semantic health search engines, which are:

1. None of the search engines concerns health service retrieval. The existing semantic health search engines are all designed for searching health information/documents. None of the projects pays attention to assisting service requesters to search health service advertisements and health service provider profiles over the Internet.

2. None of the projects is concerned with semantically modeling health service domain knowledge in Digital Health Ecosystems. Although there are several health ontologies designed to provide shared vocabularies for information disambiguation, none of the ontologies contributes to the service field, owing to the fact that heath service knowledge is different from health knowledge.

\subsection{Semantic Service Matchmakers}

Within the discipline of service computing, semantic web technologies are widely used in the area of web services. In this area, semantic web technologies are used to add semantics to web service description languages such as WSDL. As a result, the performance of service discovery and service matchmaking can be enhanced by semanticizing the web service descriptions. Service matchmaking refers to the process of matching between service requests and similar service advertisements in the web service environment $[18,33]$. The reason for using semantic web technologies is that the aforementioned web service description languages can specify the operations and data structure, but cannot specify the meaning and semantic constraints of data involved in web services, which may generate problems during the matchmaking process [41]. Therefore, a new form of web services has emerged, known as semantic web services. Semantic web services empower web services with a new level of interoperability, by means of adding DARPA agent markup language for services (DAML-S), Web Ontology Language for services (OWL-S) or Web Service Modeling Ontology (WSMO)-encoded semantic annotations to specific business functionalities, with the goal of enabling automatic service discovery, manipulation and composition [19]. Meanwhile, researchers have designed many matchmaking mechanisms for semantic web services.

The semantic service matchmakers designed for DAML-S-encoded semantic web services are described as follows:

Kawamura et al. [30] designed a Semantic Service Matchmaker (SSM) in order to search services in the UDDI Business Registry. The services are coded with the Web Service Semantic Profile (WSSP), inspired by the DAML-S Service Profile, in order to encode semantic information 
into the WSDL-encoded services. A constraint filter is then used to determine whether a service request can be subsumed by a service.

Li and Horrocks [34] introduced the use of DAML+OIL and the DAML-S ontology in order to semantically represent the descriptions of services, service advertisements and service requests. A matching algorithm is developed to match service advertisements with service requests. Similarly, Li et al. [35] proposed an ontology-based matchmaking approach for web services in e-markets. This approach contains a DAML-based domain ontology and a decision-making, model-based semantic matchmaking mechanism.

Kun et al. [32] proposed a methodology for agent service matchmaking, which matches between agent services' functional properties and non-functional properties. The functional-propertymatching is realized by building domain ontologies for describing agent services' profiles and using a semantic similarity model to compute the similarity values between agents' service profiles based on the domain ontologies. The similarity values are then integrated with the non-functionalproperty-matching result, which generates the eventual matching values between agent services.

The semantic service matchmakers designed for OWL-S-encoded semantic web services are described as follows:

Kluch et al. [31] introduced a hybrid semantic web service matchmaker for OWL-S services (OWLS-MX). The hybrid approach exploits both the logic-based and non-logic-based approaches for OWL-S service profile signature matching. Initially, the OWLS-MX computes the extent of semantic matching between service advertisements and requests by applying a combination of the logic-based and non-logic-based filters. Next, the OWLS-MX employs a matchmaker ontology to denote the filtered OWL-S service advertisements and requests, followed by a generic matchmaking algorithm for computing the similarity between the services' advertisements and the requests.

Bianchini et al. $[4,5]$ proposed an ontology-based hybrid matchmaking approach. First of all, a service can be described by the conjunction of OWL-Description Logic (DL)-based concepts from a service ontology. Thus, the OWL-DL-based classification is used to precisely establish the kind of matching between a request and an advertised service, by means of deducing their relationship in the ontology. The similarity values between the request and its partially matched services are then computed and ranked. Similar to Bianchini et al.'s approach, Chiat et al. [10] made use of DL to match semantic web services with service requests.

Sriharee and Senivongse [40] defined a series of service profile-based mechanisms for semantic web service matchmaking. They built an OWL-encoded upper ontology for modeling the profile of web services. The profile integrates the information with regard to the attributes, capability, structure, behavior and constraints of web services. Based on the integrated web service profile, they provided multiple matching criteria for web service matchmaking. The matched web services can also be ranked based upon the integrative values from all matchmaking criteria.

Fenza et al. [19] designed a hybrid approach which combines an agent-based paradigm and fuzzy modeling for semantic web service matchmaking. They used OWL-S and fuzzy multiset to represent service profiles, which forms a knowledge layer for web services. Based on the knowledge layer, they designed an agents layer which comprises broker agents and advertiser agents. The broker agents employ the fuzzy matchmaking mechanism to match with the advertiser agents in order to discover appropriate services for service requests.

Dogac et al. [13] proposed an Artemis project aiming at bringing semantics to web services in the health informatics domain. They used OWL to describe the semantics of web services' functionalities and exchanging messages by linking them to healthcare information standards through standard bodies such as HL7, CEN TC251, ISO, TC215 and GEHR.

Apart from the web service environment, a series of semantic service matchmakers have been developed for retrieving resources in the Grid environment. Tangmunarunkit et al. [44] proposed such a semantic service matchmaker, which is comprised of ontologies, domain background knowledge and matchmaking rules. There are three categories of ontologies: 1) resource ontologies for describing resources on the Grid; 2) resource request ontologies for describing requests; 3) policy ontologies for describing the authorization and usage policy of resources. In addition, matchmaking rules are designed with the ontologies and the ontology-annotated domain background knowledge, in order to match resource providers with requests. Analogously, Harth et al. [23] designed an ontology-based matchmaker for performing resource selection on the Grid, by using terms defined in ontologies to form a loose coupling between resources and request descriptions. In addition, Ludwig et al. [36] proposed a semantic service matchmaker for the Grid environment, which enables the matching between service requests and semantic descriptions of service properties.

The limitations of the above approaches can be concluded as follows: 
1. None of the approaches focuses on generic health service matchmaking in Digital Health Ecosystems. It can be observed that all of the existing methodologies focus only on WSDL-described web service matchmaking and ignore arbitrary language-described generic service information (including health service information) over the Internet.

2. Similar to the issue observed in Section 2.1, none of the approaches contributes to semantically modeling health service domain knowledge for Digital Health Ecosystems.

\section{Solution Overview}

In order to resolve the issues presented in the introduction and the literature review, in this paper, we propose a framework for a semantic service matchmaker, taking into account the heterogeneous and ambiguous nature of services in Digital Health Ecosystems. The system overview of this framework is shown in Fig. 1. This framework is extended from our previous work in the transport service domain [17], and is dedicated to four areas of Digital Health Ecosystems as follows:

- Health service domain knowledge modeling. To address the issue of the absence of health service domain knowledge modeling, we define a health service ontology in order to define the abstract-level service domain knowledge in Digital Health Ecosystems. This health service ontology is stored in the Health Service Knowledge Base displayed in Fig. 1. This ontology can be employed in the areas of health service information disambiguation, health service query disambiguation and health service quality evaluation. In Section 4, we provide an overview of the health service ontology.

- Online health service information disambiguation. In order to resolve the issue of heath service information ambiguity over the Internet, we define a unified health service metadata schema, which includes the schema for encapsulating and standardizing health service advertisement and health service provider profile information. In terms of the schema, a framework of a semantic focused crawler is proposed by us in order to retrieve health service information from webpages, generate health service metadata from the fetched documents and then store them in the Health Service Metadata Base in Fig. 1. In addition, the semantic focused crawler is able to annotate the generated health service metadata with the concepts from the health service ontology. We present the technical details of the semantic focused crawler in [15]. In Section 4, we present the health service metadata schema.

- Health service query disambiguation. The main objective of this paper is to present a framework of a semantic service matchmaker, in order to help service requesters to disambiguate their service queries and denote their query intentions. In Section 5.1, we reveal a restricted view-based service matchmaking module for service requesters to precisely search requested service advertisements, by incorporating the technology of the view-based search and the semantic service matchmaking.

- Health service quality evaluation. In Section 5.3, to address the trust issue of online health service advertisements, we present a QoS evaluation and ranking methodology, which allows service requesters to domain-dependently evaluate the quality of health services after completing transactions with the services. Meanwhile, the QoS evaluation criteria of each domain is determined by a corresponding concept from the health service ontology. The evaluation data are then stored in the Health Service Quality Database displayed in Fig. 1, and employed for health service ranking. 


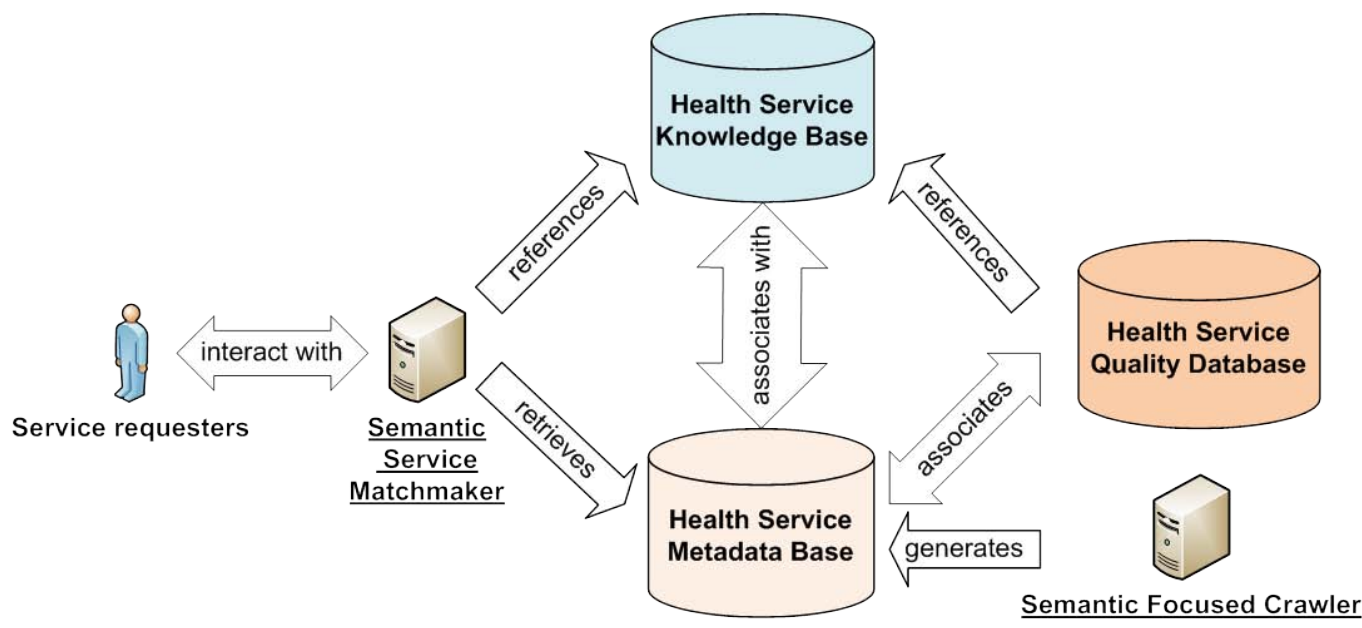

Figure 1. System overview of the proposed solution

\section{$4 \quad$ Health Service Ontology and Health Service Metadata Schema}

As mentioned previously, a health service ontology can be used for modeling high-level service domain knowledge in Digital Health Ecosystems, with the purpose of disambiguating health service information over the Internet, assisting service requesters to disambiguate their service queries, and domain-dependently evaluating and ranking the quality of retrieved service advertisements. However, currently no such ontology is available. Therefore, we present a service ontology in this subsection.

We define the health service ontology from the perspective of concept hierarchy and ontology concepts.

The health service concept hierarchy is a four-tier structure with 218 concepts, in which the lower level concepts are the specification of the upper level concepts. The root concept defines the boundary of the general health service domain and the properties of a generic health service. The second tier concepts define the six health service subdomains, which are allied health service, dentistry service, medicine service, nursing service, pathological and clinical laboratory service, and hospital and clinic service. The third and fourth tier concepts are the further specification of the subdomain concepts, which also inherit the properties from their superclass and also have their own domain-specific properties. It is noted that the health service ontology is designed by referring to the relevant knowledge from Wikipedia (http://en.wikipedia.org/wiki/), Open Directory Project (ODP) (http://www.dmoz.org), and hundreds of international health service providers' websites. The abbreviated view of the health service concept hierarchy can be found from Fig. 2.

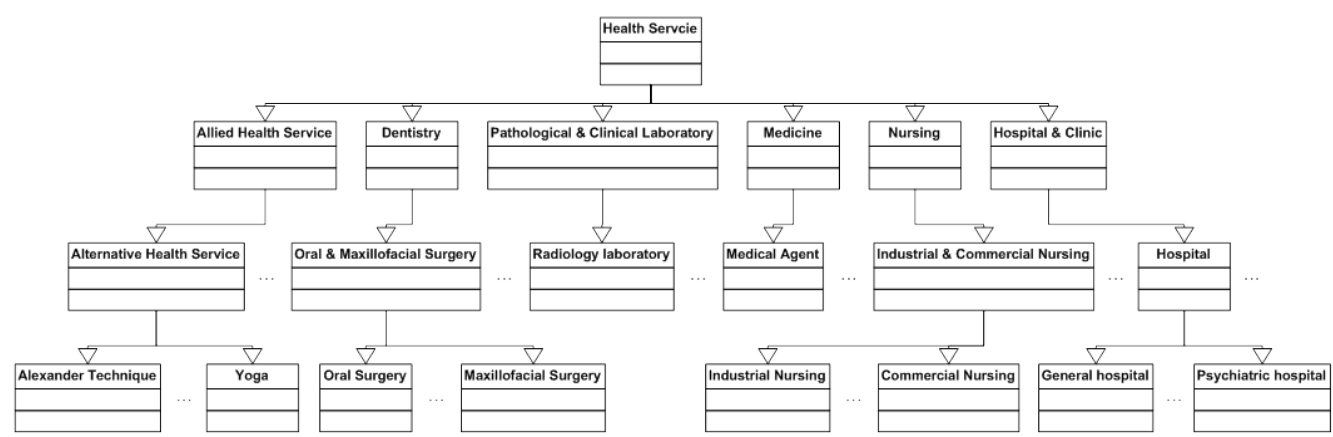

Figure 2. Abbreviated view of the health service concept hierarchy

Whereas each concept in the health service concept hierarchy inherits the properties from its parent concept, because of the knowledge differences among health service subdomains, each concept should have domain-specific properties to outline its speciality. To allow the domain speciality, we define a property of conceptDescription, which is an extended property that can be an arbitrary amount. The conceptDescription property(s) contains domain-specific information describing a health service concept, which is used for the forthcoming query-concept similarity 
computation introduced in Section 5. Additionally, to enable a health service metadata to be annotated by at least one health service concept, we design the property of linkedMetadata, which is used to store the URI(s) of metadata associated with a concept. The abbreviated view of a generic health service concept schema encoded in RDFS can be viewed below:

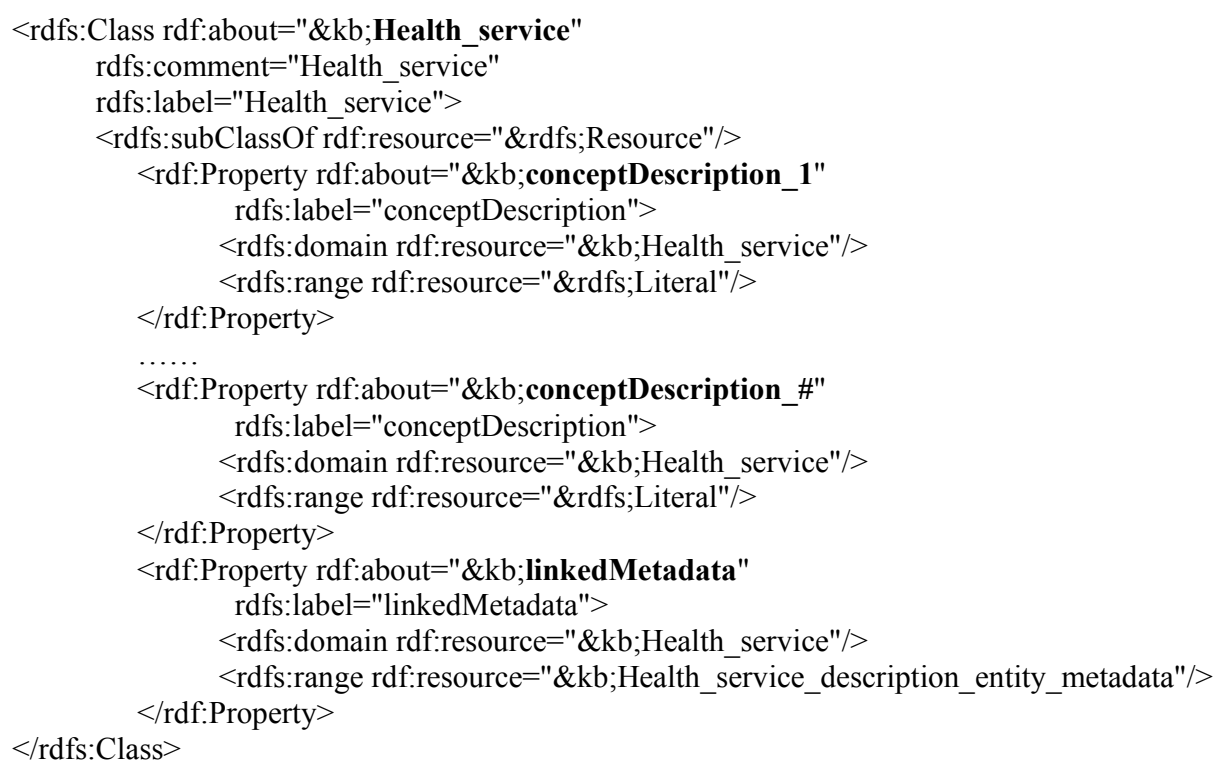

As mentioned in Section 3, the semantic-focused crawler makes use of a unified health service metadata schema in order to build health service metadata. The health service metadata schema consists of two-double layers of schemas, which are the Health Service Description Entity (HSDE) schema on the top layer and the Health Service Provider (HSP) schema on the bottom layer. Of them, the HSDE schema is used to build an HSDE metadata that describes a health service advertisement, and the HSP schema is used to build an HSP metadata that describes a service provider profile [15]. To illustrate the schemas in detail, we display an example of the health service metadata in Fig. 3. It can be found that there are links between the HSDE schema and the HSP schema, by which a HSP metadata may associate to at least one HSDE metadata (one example of the link is "Health risk assessment consultants" under the label "Other Services" in Fig. 3). Moreover, the HSDE metadata has a linkedConcepts property, which is the inverse property of the linkedMetadata property of a health service ontology concept. 


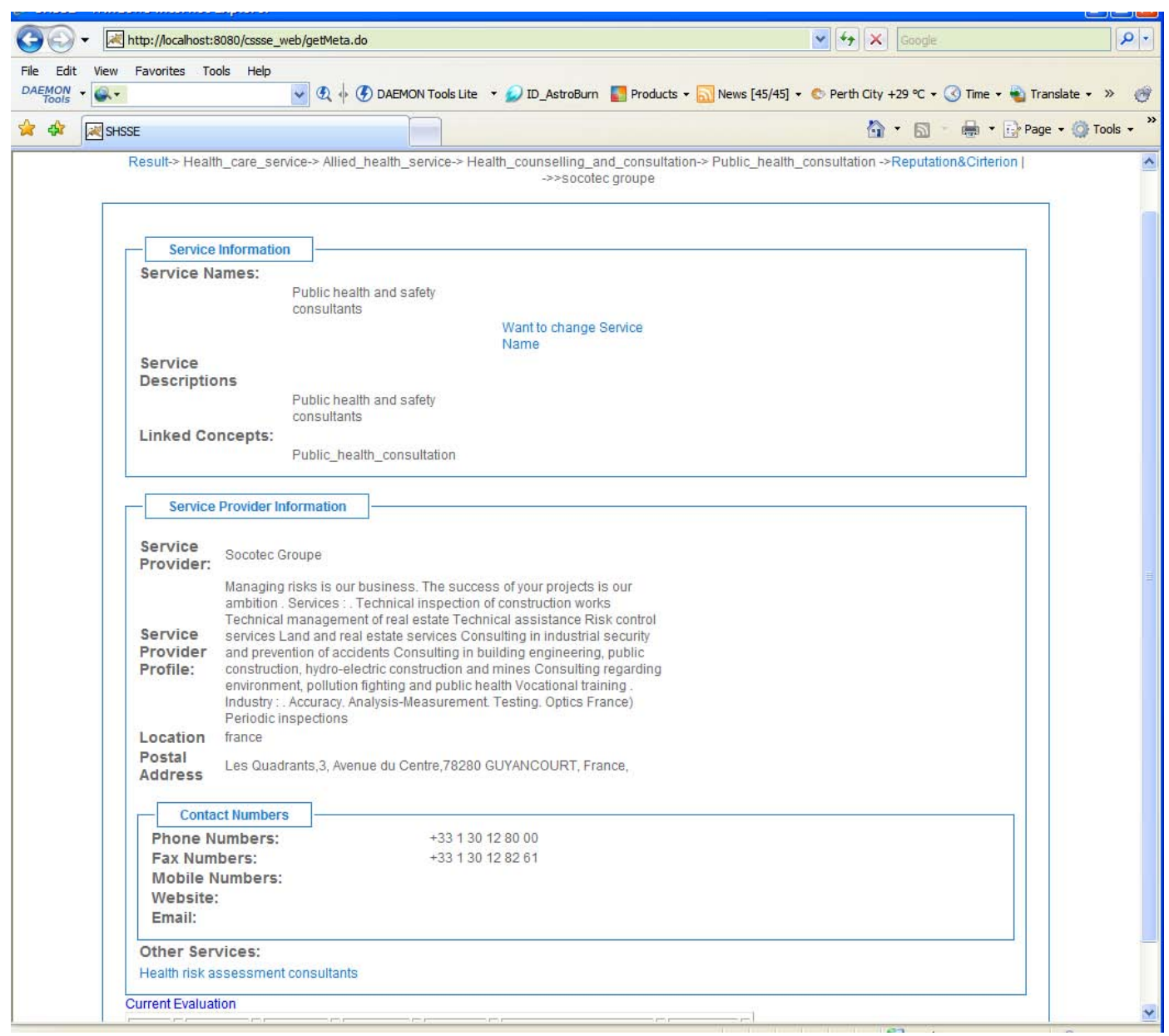

Figure 3. Example of a health service metadata

\section{Semantic Service Matchmaker}

In this section, we reveal the technical details of the proposed semantic service matchmaker, including its system workflow, the involved algorithm and the QoS-based service evaluation and ranking methodology.

\subsection{System Workflow}

The goal of the proposed framework is to disambiguate service requesters' service queries, and to assist them to retrieve the service information which can precisely match their query intentions. To realize this goal, we take advantage of the idea of facet (or called view-based) search from the semantic search field and the idea of semantic service matchmaking from the web service field. Faceted search refers to the search technology that provides a set of predefined, high-level categories called facets for result categorization [20]. From our literature view in Section 2.2, it can be found that the semantic service matchmaking approaches use ontology concepts to annotate the functionalities involved in both service requests and service advertisements and then matches between annotated service requests and service advertisements. On the one hand, we design the semantic focused crawler, the health service ontology and the health service metadata schema to implement the task of service advertisement annotation; moreover, we design a restricted viewbased search paradigm to help service requesters to annotate their service queries in terms of the health service ontology concepts. The system workflow of the semantic service matchmaker is shown in Fig. 4. 


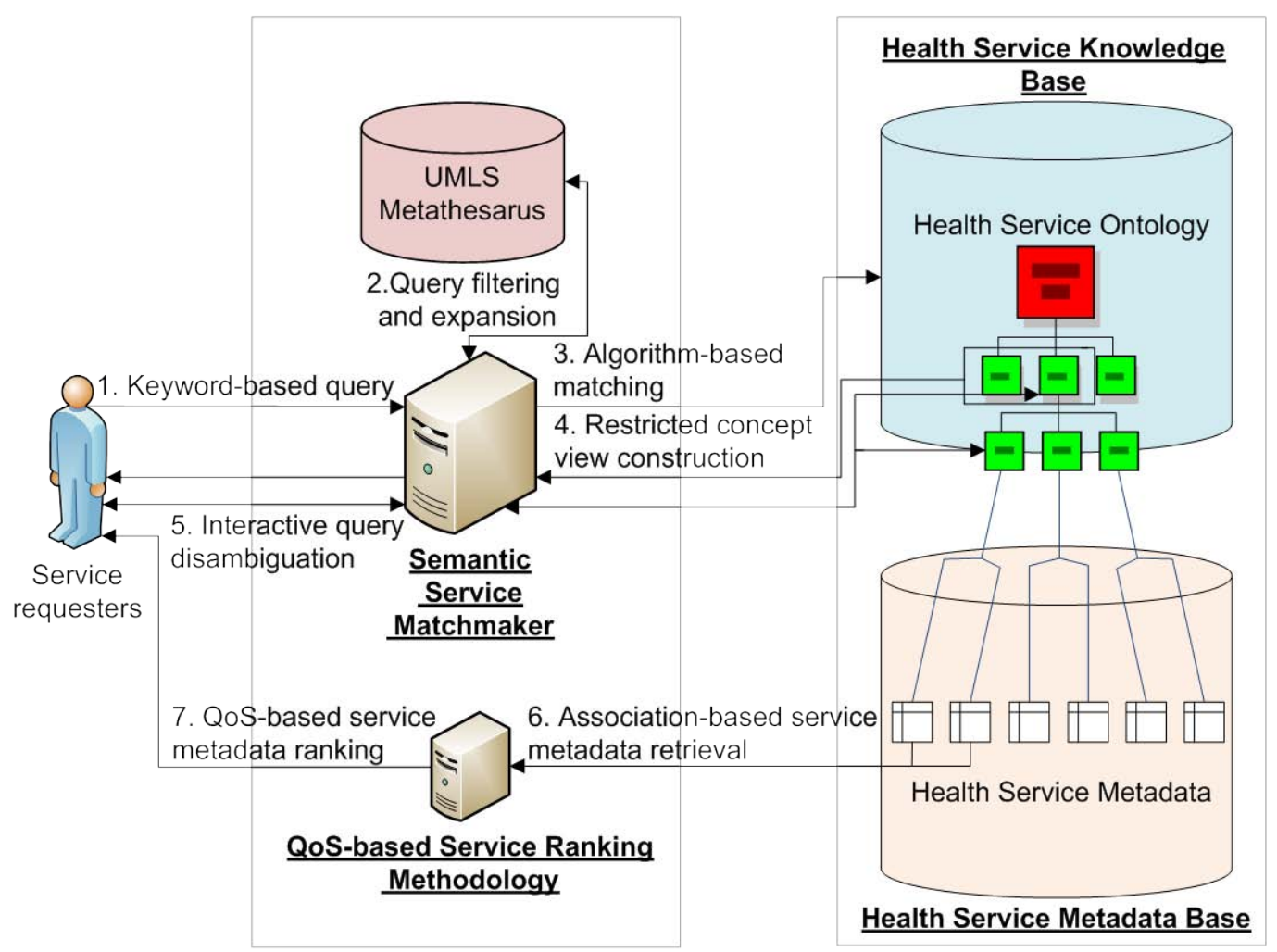

Figure 4. System workflow of the semantic service matchmaker

Next, we explain the whole semantic service matchmaking process step by step as follows:

Step 1. Keyword-based query. As most users prefer keyword-based search interfaces (e.g. Google ${ }^{\mathrm{TM}}$ ), we premise that the inputs of our system, namely the service requests, are keywordbased queries, resulting from a survey which indicates that the average query length for most of the web search engines is around 2.3 words [24]. Consequently, considering the processing time, we do not need to specially employ natural language processing (NLP) programs to parse and analyze service queries.

Step 2. Query filtering and expansion. Instead of the NLP, we employ the UMLS metathesaurus (http://www.nlm.nih.gov/research/umls/) to implement the task of query processing and expansion. By sending all words of a service query to the UMLS metathesaurus, for the valid words, the thesaurus will return the synonyms of the words; for the invalid words, the thesaurus will return error messages. Hence, within the query, the valid words will be expanded with its synonyms and the invalid words will be filtered.

Step 3. Algorithm-based matching. After receiving a processed query from the UMLS metathesaurus, the search engine will match the query with each concept within the health service ontology. This query-concept matching is realized by an algorithm, which is introduced in Section 5.2. The algorithm is able to compute the similarity value between the query and each concept.

Step 4. Restricted concept view construction. The retrieved concepts matched with the query will be ranked according to their similarity values and grouped as a view as well as being displayed in front of service requesters.

Step 5. Interactive query disambiguation. The query disambiguation process is realized by a restricted view-based approach, which consists of the subsequent interactions:

Step 5.1. A service requester needs to choose a service concept from the returned concept view, in order to find a concept that can best denote his/her query intention.

Step 5.2. If the selected concept is an upper-level concept in the health service ontology, it will unfold all its subconcepts from the ontology, for the user's further denotation (concept selection). Steps 5.1 and 5.2 are an iterative process unless the service requester selects a concrete concept.

Step 6. Association-based service metadata retrieval. If a service requester chooses a bottomlevel service concept, the search engine will return all its associated metadata from the Health Service Metadata Base by retrieving the linkedMetadata property of the concept.

Step 7. QoS-based service metadata ranking. The retrieved health service metadata will be ranked by a QoS-based service ranking methodology and displayed to a service requester. The 
service requester can then select a metadata from the list and find its detailed information (e.g. Fig. 3). The QoS-based service ranking methodology is introduced in Section 5.3.

There are two main advantages with the restricted view-based approach, which are described as follows:

1. This approach does not purely match concepts with a query based on their similarity values with the query. On the contrary, it displays all the subclasses of a matched concept to a service requester, and does not omit concepts that could be relevant to the query. Moreover, this approach allows requesters' subjective perceptions towards their queries to work in the query disambiguation process, which potentially enhances users' satisfaction towards the concept selection, since users play the most decisive roles in this process.

2. The concepts comprising a view are selected based on the similarity values between the concepts and a query, which ignores the concepts irrelevant to the query, reduces the scope of user viewing, and facilitates the view-based search. This eliminates the limitation of the view-based search whereby views with large-scale options could bring users obvious inconvenience, when considering the time cost factor.

\subsection{Query-Concept Matching Algorithm}

As introduced above, the algorithm-based query-concept matching requires a mathematical model to compute the similarity values between a service query and all concepts from the health service ontology. Here, we design an Index term-based Extended Case Based Reasoning (IECBR) algorithm, which is an enhanced version of the Extended Case Based Reasoning (ECBR) algorithm proposed in our previous work [14]. By introducing the theory of index terms into the ECBR, it is expected that the IECBR algorithm will be more efficient than the ECBR algorithm. The principle of the IECBR model is to seek the maximum similarity value between a query and all conceptDescription properties of a concept. The IECBR-based query-concept matching algorithm divides the matching process into two steps as follows:

Step 1. Generating an index term list before receiving a query, by obtaining all terms from all conceptDescription properties of a concept; each conceptDescription property is then assigned with an array, in which each element corresponds to the each element of the index term list, and the weight of each element is 1 if the corresponding index term occurs in the conceptDescription property, or 0 otherwise.

Step 2. Having received a processed query from the UMLS metathesaurus, the search engine will generate an array for the query, based on the index term list. Each element of the array is assigned with a weight $\{0,0.5,1\}$ where 1 indicates that the corresponding index term occurs in the query words, 0.5 indicates that the corresponding index term occurs in the synonyms of the query words and 0 indicates not. The array of the query is then compared with the arrays of the conceptDescription properties of each concept from the health service ontology. The maximum value between the query and any conceptDescription properties of a concept is considered as the similarity value between the query and the concept.

The IECBR-based query-concept matching algorithm can be mathematically expressed as:

$$
\operatorname{sim}(q, c)=\max _{c d_{j} \in c}\left(\sum_{t_{j h} \in c d_{j}} \frac{f\left(q, t_{j h}\right)+f_{s}\left(s, t_{j h}\right)}{\sum c d_{j}}\right)
$$

with

$$
\begin{aligned}
& f\left(q, t_{j h}\right)= \begin{cases}1 & \text { if } \exists \Delta \mid\left(\forall k_{t},\left(g_{t}\left(t_{j h}\right)=g_{t}(\Delta)\right) \wedge(\Delta \in q)\right) \\
0 & \text { otherwise }\end{cases} \\
& f_{s}\left(s, t_{j h}\right)= \begin{cases}0.5 & \text { if } \exists \Delta \mid\left(\forall k_{t},\left(g_{t}\left(t_{j h}\right)=g_{t}(\Delta)\right) \wedge(\Delta \in s)\right) \\
0 & \text { otherwise }\end{cases}
\end{aligned}
$$

where $\mathrm{q}$ is a processed query, $\mathrm{c}$ is a concept, $\mathrm{cd}_{\mathrm{j}}$ is a conceptDescription property of concept $\mathrm{c}$, $\mathrm{t}_{\mathrm{jh}}$ is a term involved in $\mathrm{cd}_{\mathrm{j}}, \sum \mathrm{cd}_{\mathrm{j}}$ is the sum of the weights within the array associated with $\mathrm{cd}_{\mathrm{j}}, \Delta \mathrm{is}$ the term involved in $\mathrm{cd}_{\mathrm{j}}, \mathrm{k}_{\mathrm{t}}$ is an index term, $\mathrm{g}_{\mathrm{t}}$ is a function that returns a weight associated with $\mathrm{k}_{\mathrm{t}}$.

In a search engine, when many items are retrieved in terms of a query, a proper threshold value needs to be chosen in order to filter irrelevant items to avoid query flooding and to achieve the best performance of the search engine [7, 28, 29]. Analogously, since the health service ontology consists of hundreds of service concepts, if all the concepts from the ontology are retrieved and displayed to a service requester, regardless of whether or not it is relevant to his/her query 
intention, the service requester will easily get confused by receiving too many results. Therefore, we need to configure a threshold value to filter irrelevant service concepts for a service query based on the similarity values between queries and concepts. Moreover, upon the threshold value, the proposed semantic service matchmaker is supposed to be at its optimal performance. The process of the optimal threshold value selection is revealed in Section 6.3.

\subsection{QoS-based Service Evaluation and Ranking Methodology}

As mentioned previously, people always doubt the trustworthiness of a service advertisement after retrieving it from the web, especially for health services, as a result of their crucial role in sustaining species' activities in Digital Health Ecosystems. Hence, in our proposed semantic service matchmaker, when a service requester selects a health service ontology concept that could best represent his/her query intention and all the health service metadata associated to the concept are then retrieved from the Health Service Metadata Base, we should have a methodology to assess the trustworthiness of these metadata which are the encapsulation of those advertisements. In order to realize this objective, we design a set of Correlation, Commitment, Clarity and Importance (CCCI) metrics [16]. The CCCI metrics allow a service requester to evaluate the trustworthiness of a service provider in a service after the requester completes the service transaction with the provider. The evaluation is made by assigning different values to the commitment (ranging from 0 to 6), clarity (ranging from 0 to 1 ) and importance (ranging from 1 to 3 ) of QoS criteria of a service. In our framework, the QoS criteria of a service are determined by a health service ontology concept which represents a health service subdomain. If a service metadata that represents the service has an association with a service concept, the metadata can then be evaluated by the QoS criteria relevant to the concept. After a service requester assigns values to QoS criteria of a service metadata, the requester's perception of the trustworthiness of the service metadata is estimated by the CCCI metrics. Afterwards, the reputation value (ranging from 0 to 6 ) of the service provider in this service can be calculated by obtaining all past evaluation scores. This process can be mathematically expressed by the following algorithm:

$$
\begin{aligned}
\text { Reputation } & =\frac{\sum_{i=1}^{m} \text { Trustworhiness }_{\text {Eva }_{i}}}{m} \\
& =\frac{\sum_{i=1}^{m} \sum_{j=1}^{n} \operatorname{Comm}_{\text {Eva }_{i}, \text { Criterion }_{j}} \times \text { Clar }_{\text {Eva }_{i}, \text { Criterion }_{j}} \times \operatorname{Imp}_{\text {Eva }_{i}, \text { Criterion }_{j}}}{m \bullet \sum_{i=1}^{m} \sum_{j=1}^{n} \operatorname{MAComm}_{\text {Eva }_{i}, \text { Criterion }_{j}} \times \operatorname{Clar}_{\text {Eva }_{i}, \text { Criterion }_{j}} \times \operatorname{Imp}_{\text {Eva }_{i}, \text { Criterion }_{j}}}
\end{aligned}
$$

where $\mathrm{m}$ is the number of evaluated service requesters to a service provided by a service provider, $\mathrm{n}$ is the number of QoS evaluation criteria under a service concept associating to the service, and MAComm (MAComm $=6)$ is the mutually agreed commitment value for each criterion.

Apart from ranking service metadata based on their service providers' reputation values, we also consider the impact of service requesters' preference on particular criteria for the ranking. Thus, we allow a service requester to rank service metadata based on their values under each QoS criterion by means of the following algorithm:

$$
\text { ActualBehaviour }_{\text {Criterion }_{j}}=\frac{\sum_{i=1}^{m} \operatorname{Comm}_{\text {Eva }_{i}, \text { Criterion }_{j}}}{m}
$$

where ActualBehaviour is the average commitment value (ranging from 0 to 6) of a QoS criterion $\mathrm{j}$ assigned by past service requesters, $\mathrm{m}$ is the number of evaluators.

It is important to note that a service metadata may have associations to more than one service concepts in our framework, which means that a service represented by the service metadata may be relevant to more than one service subdomains. As different subdomains may have different QoS criteria and evaluators, the service provider who provides the service may have different reputation values for this service in different subdomains. This is close to the fact that a service provided by a service provider could be evaluated differently based on different industry standards or by different evaluators in the real world. 
According to Hevner et al. [25]'s theory, the evaluation of our semantic service matchmaking framework can be divided into two phases: 1) evaluating the proposed functions of the framework by means of the functional testing approach; 2) evaluating the performance of the framework by means of the simulation approach.

\subsection{Functional Testing}

Functional testing is an evaluation approach for design science, by which system interfaces are executed to discover the failure and defects within their functions [25]. The major contribution of this paper is a framework of a semantic service matchmaker for Digital Health Ecosystems. In order to validate the framework, we need to implement a prototype, and test the primary functions of the prototype though a use scenario.

The whole system implementation can be divided into two subtasks: 1) constructing the Health Service Knowledge Base and the Health Service Metadata Base; 2) implementing the prototype of the semantic service matchmaker.

For the first subtask, we build the Health Service Knowledge Base by means of the RDF API of Jena. The health service ontology is annotated in RDFS and RDF. In order to create the Health Service Metadata Base, we employ a semantic focused crawler designed in our previous work [15]. We choose the Australian Yellowpages ${ }^{\circledR}$ website (http://www.yellowpages.com.au) as the data source for the health service metadata collection. The crawler extracts health service information from business webpages in this website, generates RDF-encoded health service metadata based on the extracted information, and annotates the metadata with health service ontology concepts. The annotated health service metadata are then stored in the metadata base.

For the second subtask, in order to validate the proposed framework, we have implemented a prototype of the framework - a Semantic Health Service Search Engine (SHSSE), by means of Java Server Pages (JSP), JavaScript, Java Servlet, and Asynchronous JavaScript and XML (AJAX). This prototype is built upon a semantic service platform implemented in our previous work, which can be referenced from [17].

After the prototype implementation, we start to evaluate the four functions proposed in our framework in Section 3. It needs to be noted that in our previous work [15], we validated the function of online health service information disambiguation, namely the framework of a semantic focused crawler; in our previous work [17], we validated the function of QoS-based service evaluation, namely the CCCI metrics, by the functional testing approach. Therefore, by means of the following use scenario, we will evaluate the remaining functions.

In this use scenario, we premise that a service requester who lives in Western Australia (a state of Australia) requests a drug and alcohol counseling service in the local area (but $\mathrm{s} / \mathrm{he}$ does not have enough knowledge to compose the correct query words "drug and alcohol counseling"). As there are many available services in that area, the requester attempts to find out information about the overall quality of these services. Moreover, the requester may intend to make a service selection by assigning greater preference to certain criteria.

The user requirements in the above scenario encapsulate three non-evaluated functions of the semantic service matchmaker stated in Section 3 as follows:

1. Health service domain knowledge provision - providing the service requester enough health service domain knowledge about his/her service request.

2. Health service query disambiguation - helping the service requester to find the service concept that can be used to properly denote his/her service request.

3. QoS-based health service ranking - helping the service requester to select a service by providing the multidimensional quality information about the proper services.

Fig. 5 depicts a health service domain knowledge provision and health service query disambiguation scenario in the SHSSE. Since adequate knowledge about drug and alcohol counseling services is lacking, the service requester instead enters the query words "Health Consultants" in the SHSSE search interface, and the search engine returns a view of concepts ranked according to their similarity values with the query. Each concept in the list is described by a descriptions property, which provides the requester with useful knowledge about the service domains that could be relevant to his/her request. If the requester clicks a concept "Health counseling and consultation" from the retrieved concept view, all its direct subconcepts from the health service ontology are then unfolded and displayed to the user to assist the requester to further denote his/her query intention. Analogously, these subconcepts are ranked according to the similarity values between the query and concepts, thereby allowing the requester to find relevant 
concepts earlier. In addition, each concept name in the ranked list is followed by a number which indicates the amount of the health service metadata linked to this concept. From the unfolded subconcepts, the requester finds the concept "Drug and alcohol counseling" which can be used for representing his/her genuine query intention.

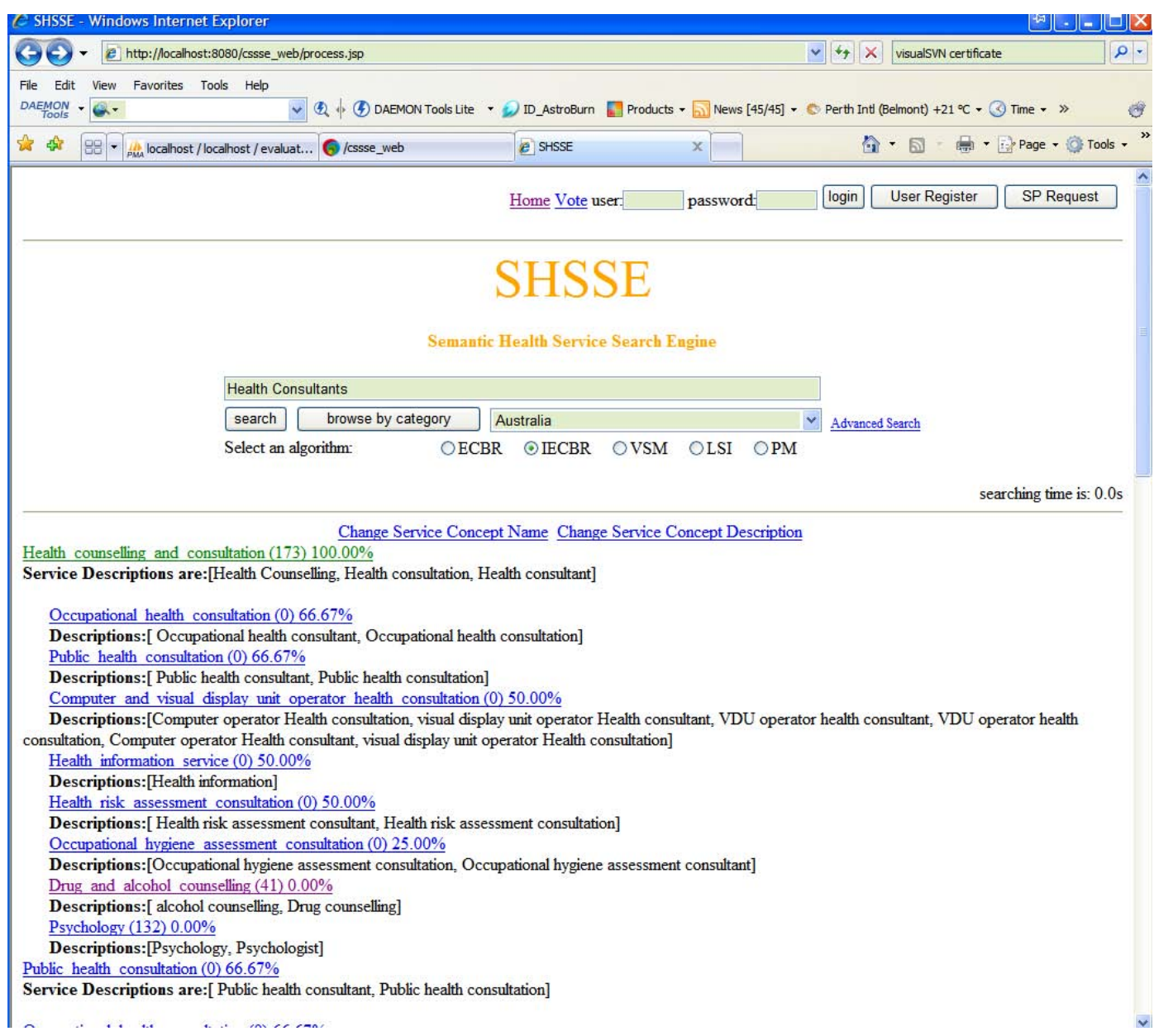

Figure 5. Screenshot of the health service domain knowledge modeling and health service query disambiguation scenario in the SHSSE

Fig. 6 reveals a QoS-based health service ranking scenario in the SHSSE. Once the service requester clicks the "Drug and alcohol counseling" concept, all the health service metadata annotated by the concept are retrieved and displayed in the interface. Moreover, if the requester chooses the state "WA", only the metadata whose providers are in WA are reserved in the interface. All the metadata are labeled by their providers' names (e.g. "Monash drink driving education" in Fig. 6), and ranked by their reputation values (values under the label "reputation" in Fig. 6) by default. The requester may rank the metadata by their ActualBehaviour values under each criterion relevant to the selected service concept (e.g. availability) or the number of evaluators (the numbers under the label "numOfEva"). In addition, the SHSSE also provides a service recommender function that recommends relevant service concepts based on the requester-selected concept (see bottom-left in Fig. 6).

From the above screenshots and descriptions, it can be summarized that the prototype basically satisfies the user requirements described in the use scenario, namely the proposed functions of the semantic matchmaking framework. Therefore, we can conclude that the semantic service matchmaking framework has preliminarily been validated by the functional testing approach. 


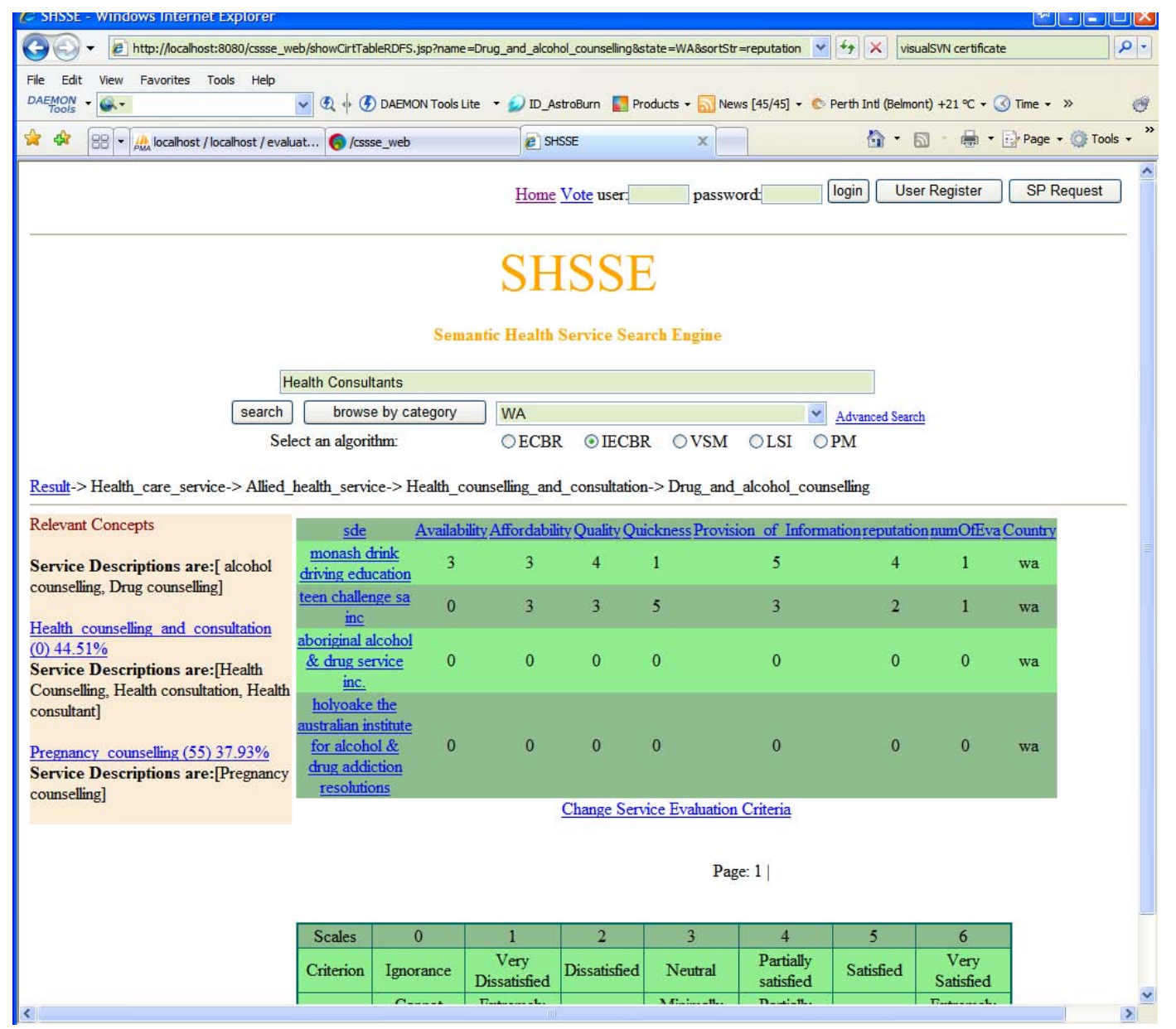

Figure 6. Screenshot of the QoS-based health service ranking scenario in the SHSSE

\subsection{Compared Algorithms in Simulation}

Simulation is an evaluation approach for testing the performance of algorithms by using artificial data [25]. By means of the simulation approach, we test the performance of the IECBR algorithm in the proposed semantic service matchmaking framework. In order to thoroughly validate the performance of the IECBR algorithm in the query-concept matching, we use the Vector Space Model (VSM), the Latent Semantic Indexing (LSI) model and the Probabilistic Model (PM), which are three classical algorithms from the traditional information retrieval field, to make a comparison with the IECBR model. In this subsection, we explain the use of the three algorithms in the queryconcept matching process in detail.

\section{Vector Space Model}

The VSM is an algebraic model for representing text documents as vectors, which is a classical model for information retrieval and indexing [39]. The implementation details of the VSM in our query-concept matching process are introduced as follows:

In the Health Service Knowledge Base, first of all, each health service ontology concept $\mathrm{c}$ is regarded as a body of plain texts comprised of conceptDescription property(s). Following that, an index term list is obtained from all the concepts in the health service ontology. Based on the index term list, each concept $\mathrm{c}$ is formed as a vector in which each element corresponds to an index term in the index term list, and the weight of each element is computed by tf-idf. Similarly, a query q can also be seen as a concept corresponding to a vector. Thus, the relevance between a concept $c_{j}$ and a query q can be calculated as the cosine of the angle between the two vectors.

$$
\operatorname{sim}\left(c_{j}, q\right)=\frac{\left|\vec{c}_{j} \cap \vec{q}\right|}{\left|\vec{c}_{j}\right| \times|\vec{q}|}=\frac{\sum_{i=1}^{t} w_{i, j} \times w_{i, q}}{\sqrt{\sum_{i=1}^{t} w_{i, j}^{2}} \times \sqrt{\sum_{i=1}^{t} w_{i, q}^{2}}}
$$


where $\vec{c}_{j}$ and $\vec{q}$ are two vectors corresponding to $\mathrm{c}_{\mathrm{j}}$ and q respectively, $\left|\vec{c}_{j}\right|$ and $|\vec{q}|$ are the norms of $\vec{c}_{j}$ and $\vec{q}$, $\mathrm{t}$ is the number of index terms in an index terms list of the health service ontology, $\mathrm{w}_{\mathrm{i}, \mathrm{j}}$ and $\mathrm{w}_{\mathrm{i}, \mathrm{q}}$ are weights of each element of $\vec{c}_{j}$ and $\vec{q}$ corresponding to each index term. Since $w_{i, j}>=0, w_{i, q}>=0, \operatorname{sim}\left(c_{j}, q\right)-[0,1]$.

\section{Latent Semantic Indexing Model}

The LSI model uses the Single Value Decomposition (SVD) technique to identity patterns within the concept-term relationships in text documents, which is used for information retrieval and indexing [11]. The implementation details of the LSI in our query-concept matching process are introduced as follows:

In the Health Service Knowledge Base, first of all, each health service ontology concept c is regarded as a body of plain texts comprised of conceptDescription property(s). Following that, an index term list is obtained from all the concepts in the health service ontology. Based on the index term list, each concept $\mathrm{c}$ is formed as an array in which each element is obtained by tf-idf, and all the concepts in the ontology are formed as a term-concept matrix A. The term-concept matrix is then decomposed by the SVD approach, which can be mathematically represented by Equation 7.

$$
A=U \Sigma V^{T}
$$

where $\mathrm{U}$ is the matrix derived from the term-to-term matrix given by $\mathrm{AA}^{\mathrm{T}}, \mathrm{V}^{\mathrm{T}}$ is the matrix derived from the transpose of the concept-to-concept matrix given by $\mathrm{A}^{\mathrm{T}} \mathrm{A}$, and $\Sigma$ is a $\mathrm{r} \times \mathrm{r}$ diagonal matrix of singular values where $r=\min (t, N)$ is the rank of $A$.

Considering that now only $\mathrm{k}$ largest singular values of $\Sigma$ are kept along with their corresponding columns in $U$ and $\mathrm{V}^{\mathrm{T}}$, the resultant $\mathrm{A}_{\mathrm{k}}$ matrix is the matrix of rank $\mathrm{k}$ which is closest to the original matrix $\mathrm{A}$ in the least square sense. This matrix is given by Equation 8 .

$$
A_{k}=U_{k} \Sigma_{k} V_{k}^{T}
$$

where $\mathrm{k}(\mathrm{k}<\mathrm{r})$ is the dimensionality of a reduced concept space.

Analogous to the concept, a query q can be formed as an index term-based array in which each element is the tf-idf weight between the query and a term from the index term list. The array can then be translated into the concept space by Equation 9, and then compared with $A_{k}$ by the cosine algorithm to calculate the similarity values of each concept, which can be represented by Equation 10 .

$$
\begin{aligned}
& q^{\prime}=\Sigma_{k}^{-1} U_{k}^{T} q \\
& \operatorname{sim}\left(c, q^{\prime}\right)=\frac{\left|A_{k} \cap q^{\prime}\right|}{\left|A_{k}\right| \times\left|q^{\prime}\right|}
\end{aligned}
$$

\section{Probabilistic Model}

The PM (also known as Binary Independence Retrieval (BIR) model) uses Bayes' theorem to estimate the similarity between queries and documents [38]. The implementation details of the PM in our query-concept matching process are introduced as follows:

In the Health Service Knowledge Base, first of all, each health service ontology concept $\mathrm{c}$ is regarded as a body of plain texts comprised of conceptDescription property(s). Following that, an index term list is obtained from all the concepts in the health service ontology. Based on the index term list, each concept $\mathrm{c}$ is formed as a vector in which each element corresponds to an index term in the index term list, and the weight of each element is 1 or 0 , in order to indicate whether or not an index term appears in the concept. Similarly, a query can be formed as a similar vector.

Given a user query $q$ and a service concept $c_{j}$ in the health service ontology, the probabilistic model tries to estimate the probability that the user will find the interesting concept. The model assumes that this probability of relevance depends only on the query and the concept representations. Furthermore, the model assumes that there is a subset of all concepts which the user prefers as the answer set for the query. Such an ideal answer set should maximize the overall probability of relevance to the user. Concepts in the ideal set are predicted to be relevant to the query, and concepts out of this set are predicted to be non-relevant. The similarity value between a concept and a query is the ratio between the possibility that the concept is relevant to the query and the possibility that the concept is non-relevant to the query. The probabilistic model is represented as follows: 


$$
\operatorname{sim}\left(c_{j}, q\right)=\frac{P\left(R \mid c_{j}\right)}{P\left(\bar{R} \mid c_{j}\right)}
$$

where $\mathrm{R}$ is the set of concepts known to be relevant to $\mathrm{q}, \bar{R}$ is the complement of $\mathrm{R}, \mathrm{P}\left(\mathrm{R} \mid \mathrm{c}_{\mathrm{j}}\right)$ is the possibility that $\mathrm{c}_{\mathrm{j}}$ is relevant to $\mathrm{q}$, and $\mathrm{P}\left(\bar{R} \mid \mathrm{c}_{\mathrm{j}}\right)$ is the possibility that that $\mathrm{c}_{\mathrm{j}}$ is non-relevant to $\mathrm{q}$.

According to Bayes' theorem, Equation 11 can be transformed to Equation 12.

$$
\operatorname{sim}\left(c_{j}, q\right)=\frac{P\left(c_{j} \mid R\right) \times P(R)}{P\left(c_{j} \mid \bar{R}\right) \times P(\bar{R})}
$$

where $\mathrm{P}\left(\mathrm{c}_{\mathrm{j}} \mid \mathrm{R}\right)$ is the possibility of selecting $\mathrm{c}_{\mathrm{j}}$ from $\mathrm{R}$, and $\mathrm{P}\left(\mathrm{c}_{\mathrm{j}} \mid \bar{R}\right)$ is the possibility of selecting $\mathrm{c}_{\mathrm{j}}$ from $\bar{R}, \mathrm{P}(\mathrm{R})$ is the possibility of selecting a concept from $\mathrm{R}$ and $\mathrm{P}(\bar{R})$ is the possibility of selecting a concept from $\bar{R}$. Since $\mathrm{P}(\mathrm{R})$ and $\mathrm{P}(\bar{R})$ both approximate the number of service concepts in the health service ontology, the similarity between $q$ and $c_{j}$ is:

$$
\operatorname{sim}\left(c_{j}, q\right) \sim \frac{P\left(c_{j} \mid R\right)}{P\left(c_{j} \mid \bar{R}\right)}
$$

Given the prerequisite of index terms independence, Equation 13 can be represented by

$$
\operatorname{sim}\left(c_{j}, q\right) \sim \frac{\left(\prod_{g_{i}\left(\bar{c}_{j}\right)=1} P\left(k_{i} \mid R\right)\right) \times\left(\prod_{g_{i}\left(\bar{c}_{j}\right)=0} P\left(\bar{k}_{i} \mid R\right)\right)}{\left(\prod_{g_{i}\left(\bar{c}_{j}\right)=1} P\left(k_{i} \mid \bar{R}\right)\right) \times\left(\prod_{g_{i}\left(\bar{c}_{j}\right)=0} P\left(\bar{k}_{i} \mid \bar{R}\right)\right)}
$$

where $\mathrm{P}\left(\mathrm{k}_{\mathrm{i}} \mid \mathrm{R}\right)$ and $\mathrm{P}\left(\mathrm{k}_{\mathrm{i}} \mid \bar{R}\right)$ are the possibility of selecting an index term $\mathrm{k}_{\mathrm{i}}$ from $\mathrm{R}$ and $\bar{R}$ respectively, $\mathrm{P}\left(\bar{k}_{i} \mid \mathrm{R}\right)$ and $\mathrm{P}\left(\bar{k}_{i} \mid \bar{R}\right)$ are the complement of $\mathrm{p}\left(\mathrm{k}_{\mathrm{i}} \mid \mathrm{R}\right)$ and $\mathrm{P}\left(\mathrm{k}_{\mathrm{i}} \mid \bar{R}\right), \quad \vec{c}_{j}$ is the vector corresponding to $\mathrm{c}_{\mathrm{j}}$, and $\mathrm{g}_{\mathrm{i}}$ is a function that returns the weight of $\vec{c}_{j}$ on the index term $\mathrm{k}_{\mathrm{i}}$.

Since $\mathrm{P}\left(\mathrm{k}_{\mathrm{i}} \mid \mathrm{R}\right)+\mathrm{P}\left(\bar{k}_{i} \mid \mathrm{R}\right)=1$, and $\mathrm{P}\left(\mathrm{k}_{\mathrm{i}} \mid \bar{R}\right)+\mathrm{P}\left(\bar{k}_{i} \mid \bar{R}\right)=1$, Equation 14 can be represented by

$$
\operatorname{sim}\left(c_{j}, q\right) \sim \sum_{i=1}^{t} w_{i, q} \times w_{i, j} \times\left(\log \frac{P\left(k_{i} \mid R\right)}{1-P\left(k_{i} \mid R\right)}+\log \frac{1-P\left(k_{i} \mid \bar{R}\right)}{P\left(k_{i} \mid \bar{R}\right)}\right)
$$

where $t$ is the number of index terms in the health service ontology, $\mathrm{w}_{\mathrm{i}, \mathrm{q}}$ is the weight of vector $\vec{q}$ on the index term $\mathrm{k}_{\mathrm{i}}$, and $\mathrm{w}_{\mathrm{i}, \mathrm{j}}$ is the weight of vector $\vec{c}_{j}$ on the index term $\mathrm{k}_{\mathrm{i}}$

Two assumptions are made for the initial value of $\mathrm{P}\left(\mathrm{k}_{\mathrm{i}} \mid \mathrm{R}\right)$ and $\mathrm{P}\left(\mathrm{k}_{\mathrm{i}} \mid \bar{R}\right)$, which are $\mathrm{P}\left(\mathrm{k}_{\mathrm{i}} \mid \mathrm{R}\right)$ is constant for all $\mathrm{k}_{\mathrm{i}}$ (equal to 0.5 ), and $\mathrm{P}\left(\mathrm{k}_{\mathrm{i}} \mid \bar{R}\right)$ is approximate to the distribution of $\mathrm{k}_{\mathrm{i}}$ in the health service ontology.

Thus, $\mathrm{P}\left(\mathrm{k}_{\mathrm{i}} \mid \mathrm{R}\right)=0.5$ and $\mathrm{P}\left(\mathrm{k}_{\mathrm{i}} \mid \bar{R}\right) \sim \mathrm{n}_{\mathrm{i}} / \mathrm{N}$, where $\mathrm{N}$ is number of service concepts in the health service ontology and $n_{i}$ is number of concepts containing $k_{i}$. Hence, Equation 15 can be converted into Equation 16.

$$
\operatorname{sim}\left(c_{j}, q\right) \sim \sum_{i=1}^{t} w_{i, q} \times w_{i, j} \times \log \left(\frac{N}{n_{i}}-1\right)
$$

Then, the performance of retrieval can be improved by the further two assumptions, which are: $\mathrm{P}\left(\mathrm{k}_{\mathrm{i}} \mid \mathrm{R}\right)$ is approximate to the distribution of $\mathrm{k}_{\mathrm{i}}$ in the retrieved concepts, and $\mathrm{P}\left(\mathrm{k}_{\mathrm{i}} \mid \bar{R}\right)$ is approximate to the distribution of $\mathrm{k}_{\mathrm{i}}$ in the non-relevant and non-retrieved concepts.

Let $\mathrm{V}$ be a subset of concepts retrieved and ranked by the probabilistic model, $\mathrm{V}_{\mathrm{i}}$ be the subset of $\mathrm{V}$ composed of the concepts in $\mathrm{V}$, which contain $\mathrm{k}_{\mathrm{i}}$, so

$$
\begin{aligned}
& P\left(k_{i} \mid R\right)=\frac{V_{i}}{V} \\
& P\left(k_{i} \mid \bar{R}\right)=\frac{n_{i}-V_{i}}{N-V}
\end{aligned}
$$

Hence, Equation 15 can be represented by 


$$
\operatorname{sim}\left(c_{j}, q\right) \sim \sum_{i=1}^{t} w_{i, q} \times w_{i, j} \times\left(\log \frac{V_{i}}{V-V_{i}}+\log \left(\frac{N-V}{n_{i}-V_{i}}-1\right)\right)
$$

\subsection{Simulation}

In Section 6.1, we utilize the method of functional testing to validate the whole system framework. Based on the prototype and user scenario introduced above, it can be stated that all the proposed functions are realized in our SHSSE system. In order to evaluate our framework, we simulate the IECBR algorithm in the SHSSE framework and compare its performance with a similar search engine and several information retrieval algorithms. The comparison will be made based on seven performance indicators from the information retrieval field, which are precision, mean average precision, recall, F-measure $(\beta=1)$ and F-measure $(\beta=2)$, fallout and response time. Here we divide the simulation task into three subtasks as follows:

1. Algorithm simulation and optimal threshold value selection - simulating the IECBR algorithm and the selected information retrieval algorithms in the SHSSE system. It needs to be noted that we switch off the function of the UMLS metathesaurus in the SHSSE when we simulate the four algorithms since we find that the UMLS metathesaurus obviously affects the efficiency of the matchmaking negatively and we want to obtain the original performance data of the algorithms without the aid of the thesaurus. In addition, as introduced in Section 5.2, after the similarity values between a query and all concepts have been obtained from an algorithm, a threshold value needs to be configured to select the most similar concepts by filtering the concepts with lower similarity values. It is obvious that the performance of the algorithms relies on the threshold value selection. Hence, we need to determine the appropriate threshold value for the optimal performance of each algorithm.

2. Algorithm comparison - evaluating the IECBR algorithm utilized in the query-concept matching by comparing the optimal performance between the IECBR algorithm and the selected algorithms.

3. Search engine comparison - evaluating the semantic service matchmaking framework by comparing the performance between the SHSSE system and a similar search engine. Here we choose a commercial search engine - the business type search in Australian Yellowpages ${ }^{\circledR}$ website (abbreviated as Yellowpages ${ }^{\circledR}$ or YP) as the object for comparison, considering that the Yellowpages ${ }^{\circledR}$ also provides the function of health service matchmaking and our semantic focused crawler uses the Yellowpages ${ }^{\circledR}$ as the data source for generating health service metadata.

\section{Subtask 1: Algorithm simulation and optimal threshold value selection}

In order to accomplish the three tasks above, we make up 100 queries which basically cover all areas in the health service domain. We then enter the 100 queries into the Yellowpages ${ }^{\circledR}$ and the SHSSE system respectively employing the IECBR, VSM, LSI and PM algorithms. As the data ranges of the four algorithms on similarity values are all within $[0,1]$, we start the threshold value at 0 , and increase it to 0.95 with a 0.05 increment at each time. We then obtain the performance data from the four algorithms at each time of the threshold value variation. Subsequently, since Fmeasure $(\beta=1)$ and F-measure $(\beta=2)$ are two aggregated indicators in the seven performance indicators, we regard them as the decisive factors for the optimal threshold value selection for each algorithm.

Fig. 7 reveals the performance of the four algorithms on F-measure $(\beta=1)$. As the harmonic mean of precision and recall, the performance of the four algorithms on F-measure $(\beta=1)$ is influenced by the factors of precision and recall. We find that both of the curves of the VSM and the LSI show an up-down trend along with the increasing threshold values. This is because the variation ranges of their precisions are greater than the variation ranges of their recall in the prophase, and are smaller in the anaphase. In contrast, because of the relative stability of the IECBR and the PM at higher threshold values, the two algorithms remain at a higher level in the anaphase. From the perspective of F-measure $(\beta=1)$, the optimal threshold value for the IECBR is 0.75 , for the VSM is 0.4 , for the LSI is 0.45 and for the PM is 0.9 . 


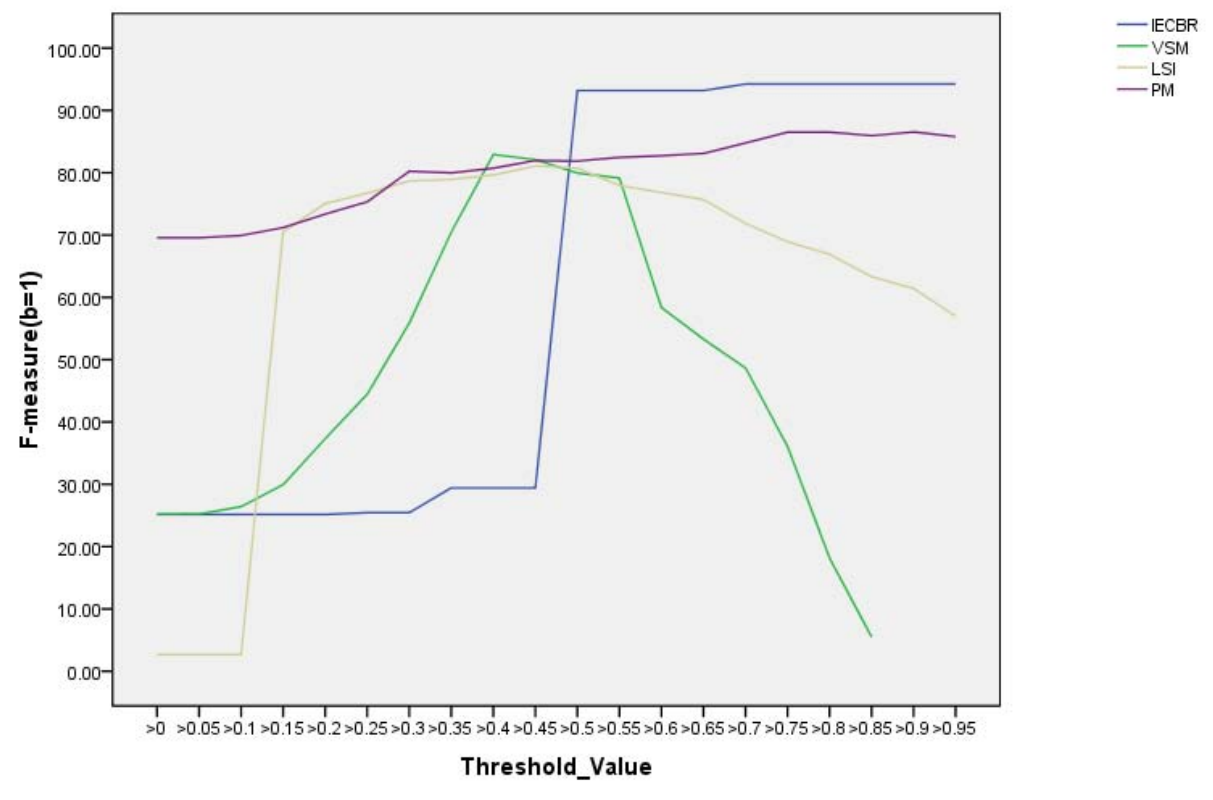

Figure 7. Comparison of the four algorithms on F-Measure $(\beta=1)$

Fig. 8 depicts the variation of the four algorithms on F-measure $(\beta=2)$. In F-measure $(\beta=2)$, the weight on recall is twice the weight on precision, which is close to the fact that most search engines are more concerned with recall other than precision, as a result of most users' purposes in obtaining information [42]. Therefore, the influence of recall on the performance of the VSM and the LSI on this indicator is more obvious than that on F-measure $(\beta=1)$. In comparison, the IECBR and the VSM remain at a higher level in the anaphase. From the perspective of F-measure $(\beta=2)$, the optimal threshold value for the IECBR is 0.5 , for the VSM is 0.4 , for the LSI is 0.2 and for the PM is 0.3 .

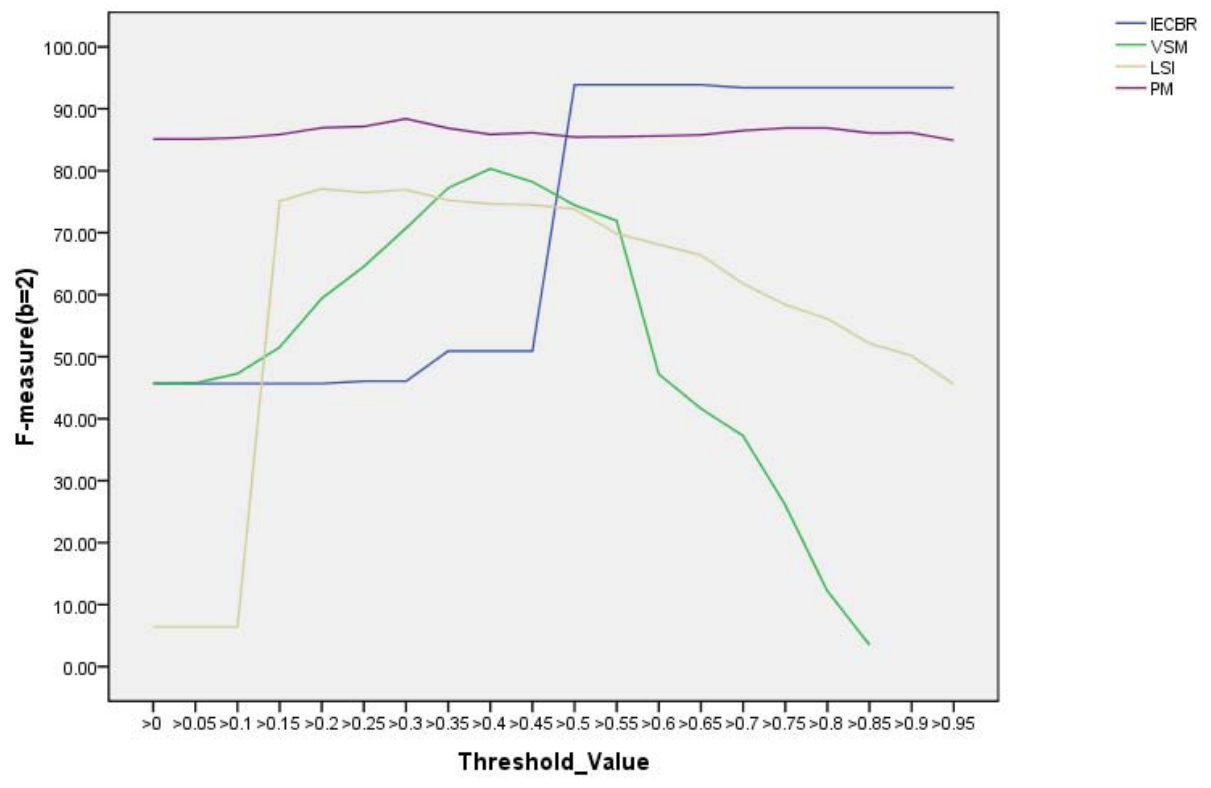

Figure 8. Comparison of the four algorithms on F-Measure $(\beta=2)$

Subtask 2 and 3: Algorithm and Search Engine Comparison

In this subsection, we combine Subtasks 2 and 3, thereby simultaneously comparing the performance of our IECBR algorithm with the three classical information retrieval algorithms and the Yellowpages ${ }^{\circledR}$. After we obtain two groups of optimal threshold values for each algorithm in Subtask 1, we compare their performance at these optimal threshold values with the performance 
of the Yellowpages ${ }^{\circledR}$ in terms of the seven performance indicators. The comparison results are respectively shown in Table 1 and 2. It needs to be noted that the Yellowpages ${ }^{\circledR}$ does not provide an API for testing and thus, we cannot obtain its algorithm and threshold value. Because there are a huge number of non-relevant concepts in the Yellowpages ${ }^{\circledR}$, we also cannot calculate its exact fallout value.

Table 1. Comparison of the performance of the four algorithms with the Yellowpages ${ }^{\circledR}$ from the perspective of the optimal F-measure $(\beta=1)$

\begin{tabular}{lllllll}
\hline \multirow{2}{*}{ Algorithms } & Optimal & \multicolumn{2}{l}{ Mean Average } & \multicolumn{2}{l}{ F-measure } \\
threshold values Precision & Precision & Recall & $(\beta=1)$ & Fallout \\
\hline IECBR & $>0.7$ & $95.67 \%$ & $99.33 \%$ & $92.83 \%$ & $94.23 \%$ & $0.02 \%$ \\
VSM & $>0.4$ & $87.69 \%$ & $98.47 \%$ & $78.67 \%$ & $82.93 \%$ & $0.07 \%$ \\
LSI & $>0.45$ & $95.11 \%$ & $100.00 \%$ & $70.67 \%$ & $81.09 \%$ & $0.01 \%$ \\
PM & $>0.9$ & $87.28 \%$ & $98.89 \%$ & $85.83 \%$ & $86.55 \%$ & $0.07 \%$ \\
YP & N/A & $22.41 \%$ & $77.33 \%$ & $42.27 \%$ & $29.29 \%$ & N/A \\
\hline
\end{tabular}

Table 2. Comparison of the performance of the four algorithms with the Yellowpages ${ }^{\circledR}$ from the perspective of the optimal F-measure $(\beta=2)$

\begin{tabular}{lllllll}
\hline \multirow{2}{*}{ Algorithms } & Optimal & \multicolumn{2}{l}{ Mean Average } & \multicolumn{2}{l}{ F-measure } \\
threshold values Precision & Precision & Recall & $(\beta=2)$ & Fallout \\
\hline IECBR & $>0.5$ & $92.08 \%$ & $99.33 \%$ & $94.33 \%$ & $93.87 \%$ & $0.05 \%$ \\
VSM & $>0.4$ & $87.69 \%$ & $98.47 \%$ & $78.67 \%$ & $80.32 \%$ & $0.07 \%$ \\
LSI & $>0.2$ & $71.90 \%$ & $95.99 \%$ & $78.50 \%$ & $77.09 \%$ & $0.18 \%$ \\
PM & $>0.3$ & $69.52 \%$ & $95.29 \%$ & $94.83 \%$ & $88.39 \%$ & $0.32 \%$ \\
YP & N/A & $22.41 \%$ & $77.33 \%$ & $42.27 \%$ & $35.90 \%$ & N/A \\
\hline
\end{tabular}

From Table 1, it can be found that the IECBR has an overall advantage over the compared algorithms and the search engine from the perspective of F-measure $(\beta=1)$, as a result of the highest values on precision and recall. Moreover, the IECBR is the only algorithm whose precision and recall values are simultaneously above $90 \%$, which shows its comprehensive capability in information retrieval. Additionally, the fallout value of IECBR is among the lowest, which reveals its low error rate. Last but not least, it can clearly be observed that the performance data of the IECBR are several times higher than the Yellowpages ${ }^{\circledR}$.

From Table 2, it can be seen that the IECBR has the best performance from the perspective of Fmeasure $(\beta=2)$ and the IECBR is still the only algorithm whose precision and recall values are both above $90 \%$ apart from its lowest fallout value. Similarly, the performance data of the IECBR are several times higher than the Yellowpages ${ }^{\circledR}$ on all available metrics.

Fig. 9 displays the performance of the four algorithms on response time which is an important metrics for the scalability of search systems. Here, response time is calculated by averaging the time of 100 queries in our experiment. The PM stands at a solid position $(0.006 \mathrm{~s})$ followed by the IECBR $(0.012 \mathrm{~s})$, the VSM $(0.013 \mathrm{~s})$ and the LSI $(0.116 \mathrm{~s})$. The response time of the LSI is much longer than for the other algorithms due to the high computing cost of SVD. Since the Yellowpages ${ }^{\circledR}$ does not provide the function for displaying response time, we exclude it from this comparison.

We summarize the findings of this evaluation as follows:

1. From Table 1 and 2, it can be concluded that the IECBR has better performance than the three classical information retrieval algorithms, from either the research perspective (Fmeasure $(\beta=1))$ or the practical perspective $(\mathrm{F}$-measure $(\beta=2))$. This preliminarily proves the validity of the IECBR algorithm in the information retrieval field.

2. From Table 1 and 2, it can also be concluded that the performance of our proposed semantic matchmaking framework performs better than a typical health service search engine - the Yellowpages ${ }^{\circledR}$, which simultaneously proves the accuracy problem in the existing health service search engines and the possibility of our framework in solving the problem.

3. From Fig. 9, it can be concluded that our algorithm are among the lowest on the response time, which preliminarily proves the efficiency of the algorithm. 


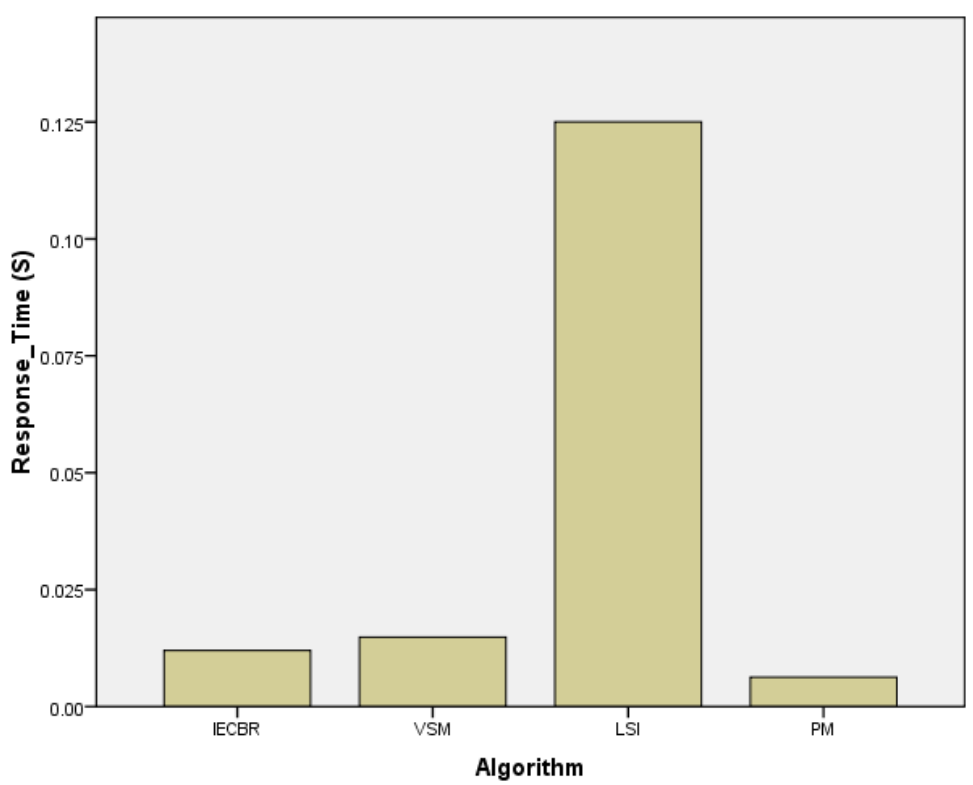

Figure 9. Comparison of the four algorithms on response time

\section{Conclusion and Future Work}

In this paper, we proposed a semantic service matchmaking framework, by taking into account the heterogeneous and ambiguous nature of service information in Digital Health Ecosystems. By means of the literature analysis, four major research issues were discovered, which are: 1) the ignorance of service domain knowledge modeling in Digital Health Ecosystems; 2) the ambiguity and heterogeneity of health service information over the Internet; 3 ) the ignorance of generic health service semantic matchmaking; and 4) the trust issue of online health service advertisements. To address the issues, we provided a framework which makes four major contributions: 1) a health service ontology for modeling health service domain knowledge in Digital Health Ecosystems; 2) a semantic focused crawler and a health service metadata schema for online health service information disambiguation; 3) a semantic service matchmaker for health service query disambiguation; and 4) a QoS-based service evaluation and ranking methodology for health service advertisement trustworthiness assessment and ranking. By means of the functional testing approach and the simulation approach, we respectively validate the semantic service matchmaking framework and its involved algorithm.

Future work is planned for the following:

- We continue to enhance our query-concept matching algorithm in order to improve its efficiency.

- Since the performance of the semantic service matchmaker relies on the quality of the health service ontology, we try to use the machine learning approach to enhance the quality of the ontology.

Acknowledgement We would like to express our appreciation to our web designer - Wei Liu who is responsible for the SHSSE system implementation and evaluation. We also would like to express our gratitude to any anonymous reviewers of our manuscript for their valuable suggestions to improve this paper.

\section{References}

[1] H. Andreassen, M. Bujnowska-Fedak, C. Chronaki, R. Dumitru, I. Pudule, S. Santana, H. Voss, R. Wynn, European citizens' use of E-health services: A study of seven countries, BMC Public Health 7 (1) (2007) 53.

[2] G. K. Berland, M. N. Elliott, L. S. Morales, J. I. Algazy, R. L. Kravitz, M. S. Broder, D. E. Kanouse, J. A. Muñoz, J.A. Puyol, M. Lara, K. E. Watkins, H. Yang, E. A. McGlynn, Health information on the Internet: Accessibility, quality, and readability in English and Spanish, Journal of American Medical Association 285 (20) (2001) 26122621.

[3] T. Berners-Lee, J. Hendler, O. Lassila, The semantic web, Scientific American Magazine (2001). 
[4] D. Bianchini, V. D. Antonellis, B. Pernici, P. Plebani, Ontology-based methodology for e-service discovery, Information Systems 31 (4) (2006) 361 - 380.

[5] D. Bianchini, V. D. Antonellis, M. Melchiori, Flexible semantic-Based service matchmaking and discovery, World Wide Web 11 (2) (2008) 227-251.

[6] A. B. Can, N. Baykal, MedicoPort: A medical search engine for all, Computer Methods and Programs in Biomedicine 86 (1) (2007) 73-86.

[7] P.-A. Champin, P. Briggs, M. Coyle, B. Smyth, Coping with noisy search experiences, Knowledge-Based Systems 23 (4) (2010) 287-294

[8] E. Chang, M. West, Digital Ecosystem - A next generation of the collaborative environment, in: Proceedings of the 8th International Conference on Information Integration and Web-based Applications \& Services (iiWAS 2006), Yogyakarta, Indonesia, 2006, pp. 3-24.

[9] E. Chang, M. West, Digital ecosystems and comparison to existing collaboration environment, WSEAS Transactions on Environment and Development 2 (11) (2006) 1396-1404.

[10] L. C. Chiat, L. Huang, J. Xie, Matchmaking for Semantic Web services, in: 2004 IEEE International Conference on Services Computing (SCC'04), Shanghai, 2004, pp. 455-458.

[11] S. C. Deerwester, S. T. Dumais, G. W. Furnas, R. A. Harshman, T. K. Landauer, K. E. Lochbaum, L. A. Streeter, Computer information retrieval using latent semantic structure. vol. 4839853, U. S. Patent, Ed. USA: Bell Communications Research, Inc., 1988.

[12] P. Dini, N. Rathbone, M. Vidal, P. Hernandez, P. Ferronato, G. Briscoe, S. Hendryx, The digital ecosystems research vision: 2010 and beyond, Creative Commons, Stanford, California, USA, 2005.

[13] A. Dogac, G. B. Laleci, S. Kirbas, Y. Kabak, S. S. Sinir, A. Yildiz, Y. Gurcan, Artemis: Deploying semantically enriched Web services in the healthcare domain, Information Systems 31 (4-5) (2006) 321-339.

[14] H. Dong, F. K. Hussain, E. Chang, A service search engine for the industrial digital ecosystems, IEEE Transactions on Industrial Electronics In Press

[15] H. Dong, F. K. Hussain, E. Chang, A framework for discovering and classifying ubiquitous services in digital health ecosystems, Journal of Computer and System Sciences In Press

[16] H. Dong, F. K. Hussain, E. Chang, A QoS-based service retrieval methodology for digital ecosystems, International Journal of Web and Grid Services 5 (3) (2009) 261-283.

[17] H. Dong, F. K. Hussain, E. Chang, A human-centered semantic service platform for the digital ecosystems environment, World Wide Web 13 (1-2) (2010) 75-103.

[18] M. Dumas, B. Benatallah, N. Russell, M. Spork, A configurable matchmaking framework for electronic marketplaces, Electronic Commerce Research and Applications 3 (1) (2004) 95-106.

[19] G. Fenza, V. Loia, S. Senatore, A hybrid approach to semantic web services matchmaking, International Journal of Approximate Reasoning 48 (3) (2008) 808-828

[20] S. Grimes, Breakthrough analysis: Two + nine types of semantic search, InformationWeek (2010).

[21] T. Gruber, A translation approach to portable ontology specifications, Knowledge Acquisition 5 (2) (1995) 199-220.

[22] M. Hadzic, A. Sidhu, Digital Health Ecosystems, in: 2nd IEEE International Conference on Digital Ecosystems and Technologies, Thailand, 2008, pp. cv-cvii.

[23] A. Harth, Y. Hey, H. Tangmunarunkity, S. Deckeryz, A semantic matchmaker service on the grid, in: WWW 2004, New York, 2004, pp. 326-327.

[24] D. Hawking, Web search engines: Part 2, Computer 39 (8) (2006) 88-90.

[25] A. R. Hevner, S. T. March, J. Park, S. Ram, Design science in information systems research, MIS Quarterly 28 (1) (2004) 75-106.

[26] M. Holi, E. Hyvönen, Fuzzy view-based semantic search, The Semantic Web - ASWC 2006, Springer-Verlag, Berlin: 2006, pp. 351-365.

[27] E. Hyvönen, K. Viljanen, O. Suominen, HealthFinland - Finnish health information on the Semantic Web, in: The 6th International Semantic Web Conference (ISWC 2007), Busan, Korea, 2007, pp. 778-791.

[28] H. Jin, X. Ning, W. Jia, H. Wu, G. Lu, Combining weights with fuzziness for intelligent semantic web search, Knowledge-Based Systems 21 (7) (2008) 655-665.

[29] S. S. Kannan, N. Ramaraj, A novel hybrid feature selection via Symmetrical Uncertainty ranking based local memetic search algorithm, Knowledge-Based Systems In Press

[30] T. Kawamura, J.-A. D. Blasio, T. Hasegawa, M. Paolucci, K. Sycara, Preliminary report of public experiment of semantic service matchmaker with UDDI business registry in: M. E. Orlowska, S. Weerawarana, M. P. Papazoglou, and J. Yang, (Eds.), ICSOC 2003, Springer-Verlag, Berlin: 2003, pp. 208-224.

[31] M. Klusch, B. Fries, K. Sycara, OWLS-MX: A hybrid Semantic Web service matchmaker for OWL-S services, Web Semantics: Science, Services and Agents on the World Wide Web 7 (2) (2009) 121-133.

[32] Z. Kun, X. Manwu, Z. Hong, X. Jian, Agent service matchmaking algorithm for autonomic element with semantic and QoS constraints, Knowledge-Based Systems 23 (2) (2010) 132-143.

[33] U. Kuster, B. Konig-Ries, M. Stern, M. Klein, DIANE - an integrated approach to automated service discovery, matchmaking and composition, in: the Sixteenth International World Wide Web Conference (WWW 2007), Banff, Alberta, Canada, 2007, pp. 1033-42.

[34] L. Li, I. Horrocks, A software framework for matchmaking based on semantic web technology, in: The Twelfth International World Wide Web Conference (WWW 2003), Budapest, Hungary, 2003, pp. 331-339.

[35] L. Li, Y. Yang, B. Wu, Ontology-based matchmaking in e-marketplace with Web Services, in: APWeb 2005, Shanghai, China, 2005, pp. 620-631.

[36] S. A. Ludwig, S. M. S. Reyhani, Introduction of semantic matchmaking to Grid computing, Journal of Parallel and Distributed Computing 65 (12) (2005) 1533-1541.

[37] F. Nachira, P. Dini, A. Nicolai, A network of digital business ecosystems for Europe: Roots, processes and perspectives, in: F. Nachira, P. Dini, A.Nicolai, M. L. Louarn, and L. R. Lèon, (Eds.), Digital Business Ecosystems, Edward Elgar Publishing, Cheltenham Glos, UK: 2007, pp. 1-20.

[38] S. E. Robertson, K. S. Jones, Relevance weighting for search terms, Journall of the American Society for Information Science 27 (3) (1976) 129-146.

[39] G. Salton, A. Wong, C. S. Yang, A vector space model for automatic indexing, Communications of the ACM 18 (11) (1975) 613-620.

[40] N. Sriharee, T. Senivongse, Matchmaking and ranking of semantic web services using integrated service profile, International Journal of Metadata, Semantics and Ontologies 1 (2) (2006) 100-118 
[41] E. Stroulia, Y. Q. Wang, Structural and semantic matching for assessing web-service similarity, International Journal of Cooperative Information Systems 14 (4) (2005) 407-437.

[42] L. T. Su, The relevance of recall and precision in user evaluation, Journal of the American Society for Information Science and Technology 45 (3) (1999) 207-217.

[43] O. Suominen, K. Viljanen, E. Hyvänen, User-centric faceted search for semantic portals, in: E. Franconi, M. Kifer, and W. May, (Eds.), The Semantic Web: Research and Applications, Springer-Verlag, Berlin, Germany: 2007, pp. 356-370.

[44] H. Tangmunarunkit, S. Decker, C. Kesselman, Ontology-based resource matching in the Grid -the Grid meets the Semantic Web in: D. Fensel, K. Sycara-Cyranski, J. Mylopoulos, and K. Sycara, (Eds.), The Semantic Web - ISWC 2003, Springer Verlag, Berlin: 2003. 